\title{
Capacitación profesoral y didáctica universitaria
}

\section{Teacher training and university didactics}

Lic. Pedro Luis González Rivera. Dr.

Universidad Politécnica Salesiana, Ecuador

Deogracia Hernández Rodríguez, Mgs.

Universidad de Pinar del Río, Cuba

Autor para correspondencia:pgonzalezr@ups.edu.ec

Fecha de recepción: 01 de Septiembre de 2016 - Fecha de aceptación: 01 de Noviembre de 2016

Resumen: Teniendo en cuenta la reconocida necesidad de la formación permanente de los docentes, el objetivo de este trabajo es reflexionar sobre la capacitación en Didáctica desde su aplicación en la práctica. Por ello, a partir de los fundamentos teóricos de la educabilidad y la educatividad, se puso en práctica un conjunto de métodos y se concibió un programa que combinara la presencialidad con el uso de recursos virtuales. De esa manera se privilegió el intercambio profesional desde las posibilidades del Aula Virtual de Aprendizaje Cooperativo (AVAC) de la universidad a través de un curso. Se pretendió posibilitar la socialización y la asimilación del contenido de forma cooperativa, prestando atención preferente a la vinculación teoría- práctica. En el plano valorativo se reafirmó la pertenencia hacia la profesión pedagógica, con independencia de las asignaturas que se dirija en el proceso de enseñanza aprendizaje. El 94 $\%$ de los participantes manifestó la incidencia positiva del curso en la transformación de la docencia. En ese ámbito se resaltó la significación de la labor didáctica para concretar los vínculos entre la ciencia y la asignatura para contribuir a la formación integral de los estudiantes, en el marco de los procesos sustantivos de la Educación Superior.

Palabras claves: capacitación profesoral; ciencias de la educación; educabilidad; educatividad didáctica universitaria

Abstract: Given the need for professorial training process in Education Science and its social significance, the objective of this work is to reflect on the training in university teaching from its application in practice. From the theoretical foundations of the educability and educatividad, an interactive program that would combine presentiality using virtual resources, was conceived through a course that would privilege the professional exchange with the possibilities of using the Virtual Classroom Cooperative Learning (AVAC) of the University. It was intended to enable socialization and assimilation cooperatively content, paying special attention to the theory linking practice. In the evaluative plane it was helped to reaffirm the sense of belonging to the teaching profession, regardless of the subjects head into the teaching-learning process. $94 \%$ of participants said the positive impact of the ongoing transformation of teaching. In this area the significance of the teaching work to finalize highlighted the links between science and subject to contribute to the integral formation of students, in the framework of the substantive processes of higher education. Key words: professorial training; science education; educability; educatividad interactive teaching university 


\section{Introducción}

En la actualidad se reconoce la necesidad del proceso de capacitación de los profesionales de la docencia en Ciencias de la Educación Superior. Organismos mundiales como la UNESCO han reiterado la importancia de la capacitación y actualización de los docentes para lograr la elevación de la calidad de educación (UNESCO, Declaración de Incheon). Los reiterados llamados a fomentar el emprendimiento como opción ante la compleja situación económica actual, serán inefectivos sin una adecuada capacitación de los educadores que forman a los profesionales universitarios (González y Hernández, 2015).

En el nivel nacional, las políticas gubernamentales enfocadas hacia el mejoramiento social prestan atención preferente al desarrollo de las capacidades educativas de los docentes en ejercicio, como vía para alcanzar peldaños superiores en la función educativa del Estado. En Ecuador, por ejemplo, las directrices del Plan Nacional del Buen Vivir (http://www.buenvivir.gob.ec/inicio) puntualizan_aspectos fundamentales de la significación social de la capacitación profesoral.

Desde el punto de vista de las exigencias sociales en el país, el Plan Nacional del Buen Vivir hace énfasis en la satisfacción de las necesidades, la consecución de una calidad de vida y muerte digna, el amar y ser amado, el florecimiento saludable de todos y todas, en paz y armonía con la naturaleza y la prolongación indefinida de las culturas humanas. Ello plantea la necesidad de avanzar en el propósito de lograr la sociedad del conocimiento, resaltando la responsabilidad de los educadores en el empoderamiento de los sectores populares, mediante el acceso cada vez más amplio a la cultura y al conocimiento en las instituciones educacionales. Para ello se requiere de programas de capacitación que coadyuven a la preparación más intensiva de los docentes universitarios para lograr la necesaria y multifacética cultura pedagógica, capaz de transformar la personalidad de los estudiantes en función de las exigencias sociales.

Por su parte, la Ley Orgánica de Educación Superior de Ecuador, en su artículo 8, establece entre los fines de la Educación Superior, que un propósito básico es la formación de personal académico y profesional. Se pretende que éste sea responsable e imbuido de conciencia ética y solidaria, capaz de contribuir al desarrollo de las instituciones de la República, a la vigencia del orden democrático. Igualmente los docentes deben estimular la participación social. A ello deben contribuir explícitamente los programas de capacitación en Educación.

Los estudios sobre la capacitación de los docentes han registrado distintas concepciones y puntos de vista en la actualidad. En ese contexto la capacitación se ha entendido como una actividad permanente, sistemática y diferenciada, a partir de la determinación de las necesidades de aprendizaje de los docentes en ejercicio para encausar adecuadamente el aprendizaje de los estudiantes. En el debate entre las diversas tendencias pedagógicas contemporáneas se insiste en la discusión sobre el papel activo del sujeto de la Educación y el rol de las nuevas tecnologías como mediadoras en el proceso de enseñanza. De esa manera, la Pedagogía y las Ciencias de la Educación se actualizan en la consideración del sistema de influencias de diversas agencias y agentes educativos como la familia, la escuela y las instituciones de la comunidad sobre el individuo. 
La reflexión sobre la función social de los profesionales de la Educación confirma la vigencia de la definición martiana sobre el acto de educar, expresada hace más de un siglo. Para el prócer latinoamericano José Martí, "Educar es depositar en cada hombre toda la obra humana que le ha antecedido: es hacer a cada hombre resumen del mundo viviente, hasta el día en que vive: es ponerlo a nivel de su tiempo, para que flote sobre él, y no dejarlo debajo de su tiempo, con lo que no podrá salir a flote; es preparar al hombre para la vida". (Martí sf, t.8, p.281)

El acercamiento a la problemática requiere del tratamiento de conceptos básicos teniendo en cuenta el uso de los recursos actuales de la información para reconocer aspectos teóricos esenciales (Padrón, González, Hernández y González, 2015).En ese sentido, la educabilidad entendida como la capacidad del individuo de ser educado (Luengo s.f) y la educatividad como la aptitud para educar (Hernández y González, 2016), adquieren nuevas dimensiones en el marco de la capacitación de los docentes. Autores como Nassif (1974) y Chávez, Suárez y Permuy (2005) aportan elementos fundamentales en la explicación de los complejos procesos educativos.

Igualmente, en este marco es significativa la incidencia fundamental de la Didáctica en el proceso formativo del docente. Para ello se consideró el acercamiento a la definición conceptual de la Didáctica, teniendo en cuenta que este constructo ha sido objeto de diversas interpretaciones. A partir de la definición comeniana de la Didáctica como artificio universal, diversas conceptualizaciones se han producido en torno a esa disciplina científica, como las expresadas por Klingberg (1972).

A finales de los años 1980 reconocidas especialistas como G. Labarrere y Valdivia (1988), señalaron que la didáctica debe ser considerada como la disciplina pedagógica que tiene en cuenta la elaboración de los principios más generales de la enseñanza. Esta concepción es aplicable a la totalidad de las asignaturas del currículo escolar, en su relación con los procesos educativos. Para dichas autoras el objeto de estudio de la Didáctica lo constituye el proceso de enseñanza-aprendizaje.

Por su parte los autores del Instituto Central de Ciencias Pedagógicas de Cuba (1999), reconocen el enfoque científico de la Didáctica y le consideran la teoría científica del proceso de enseñar y aprender, a partir de sus leyes y principios más generales. Para ello destacan la existencia de categorías básicas o componentes como los objetivos, el contenido, los métodos, los medios, las formas de organización y la evaluación, entre otras. Igualmente se considera la unidad entre educación-formación-enseñanza-aprendizaje-desarrollo y la importancia de la comunicación en este proceso. Todo ello en función de preparar al hombre para la vida, en un momento histórico social determinado (ICCP 1999). Otros autores como Addine (2004), han reafirmado el enfoque científico desde posiciones que reconocen aspectos comunes y diferencias en la definición conceptual.

Este proceso didáctico se complejiza en el nivel superior, donde la mayoría de los docentes provienen de variados sectores profesionales y su formación básica en el área pedagógica y didáctica ha sido limitada. Ello adquiere mayor relevancia ante los retos que plantea la elevación de la calidad de la formación profesional en las instituciones de Educación Superior, donde los procesos sustantivos asociados a la docencia, la investigación y la vinculación con la sociedad requieren de la profundización en el proceso educativo. 
Autores como Alzate y Arbeláez (2011), Bowden y Marton (2012) han valorado aspectos esenciales de la labor de enseñar y aprender en el nivel superior. Por su parte, Brown y Pickford (2013) han reflexionado en torno a elementos fundamentales de la enseñanza universitaria como las habilidades y las competencias.

En ese sentido, la mediación en el pensamiento pedagógico desde el Enfoque Histórico cultural vigotskiano y sus diversas dimensiones, posibilita la puesta en práctica de la capacitación de forma interactiva. Todo ello a partir del acercamiento entre docentes, facilitadores, estudiantes, familia, instituciones sociales y otros agentes y agencias educativas, con la utilización de métodos adecuados, formas de organización del proceso docente eficaces y el apoyo de medios o recursos variados.

En general se reconoce que algunas exigencias a la actividad docente en la actualidad sugieren transitar conscientemente por el prisma del análisis psicológico y pedagógico de la estructura de la actividad, centrando el proceso de enseñanza aprendizaje en el estudiante, a partir de los preceptos psicológicos de las diferencias individuales. De igual manera se plantea la necesidad de impregnarse del principio de justicia social y el humanismo, así como generar actividad intelectual productiva a partir del pensamiento divergente.

En la labor docente se valora la pertinencia de transitar por los momentos de la actividad de aprendizaje (Orientación, ejecución y control), desplegar en sistema y con carácter integrador los componentes o categorías didácticas básicas (objetivo, contenido, método, medio, evaluación y forma de organización), atender a los componentes de la formación profesional (académico laboral, investigativo y extensionista) e insertar de forma pertinente la intra, e inter y transdisciplinariedad.

Se ha considerado que la capacitación en Didáctica, apoyada en el uso de recursos de la información y tecnologías educativas, puede resultar efectiva si contribuye a elevar la cultura pedagógica del docente (García-Valcárcel, 2011). El uso eficiente de las redes internas de las instituciones universitarias puede favorecer los procesos de adquisición y consolidación de conocimientos en temas centrales de la teoría y la práctica del "artificio universal" para enseñar, como decía el padre de la Didáctica Juan Amos Comenio. La existencia de recursos tecnológicos como las aulas virtuales puede favorecer sistemas educativos basados en el aprendizaje de los alumnos de forma cooperada. Ello también puede ser factible en la capacitación didáctica de los docentes.

\section{Métodos}

En el caso de estudio, los antecedentes evidencian la necesidad de profundizar en determinados aspectos de la formación permanente de los docentes para la ejecución del proceso de enseñanza aprendizaje en las disciplinas universitarias. El diagnóstico realizado a partir del análisis de criterios de las estructuras de dirección institucional y los resultados de la evaluación profesoral, así como la observación en talleres y cursos de actualización académica, sugiere prestar atención diferenciada a la capacitación de los docentes para lograr que la clase sea más activa y desarrolladora y se realice el uso óptimo de los recursos didácticos de la asignatura. 
Se ha considerado que tales indicadores de la gestión académica contribuyen significativamente a elevar la gestión de dirección del docente en función del aprendizaje activo del alumno, como centro del proceso educativo. De esa manera se revela la naturaleza esencialmente educativa del problema en discusión, atendiendo a la conceptualización más generalizada de la Didáctica como la rama de la Pedagogía que se encarga de los complejos proceso de enseñar y aprender.

En este trabajo se ha planteado la interrogante de ¿cómo contribuir a la actualización en temas esenciales de la Didáctica de los docentes universitarios, teniendo en cuenta las necesidades de la profesión pedagógica en la actualidad? De ahí que el objetivo del trabajo se dirige a reflexionar en torno a la capacitación interactiva en Didáctica universitaria de los docentes universitarios, planteando una de las posibles soluciones. Se ha partido de la suposición de que, si se diseña un sistema de capacitación desde los componentes didácticos y principalmente la clase, entonces se lograrán mayores niveles de efectividad en el proceso de enseñanza aprendizaje.

Para ello se tuvo en cuenta un conjunto de métodos, como el análisis y la síntesis, la inducción y la deducción, así como el análisis documental y la experimentación pedagógica, entre otros. La aplicación de la experiencia abarcó un grupo de docentes con rangos de 2 a 10 años en la docencia universitaria, con formación profesional variada, en áreas no pedagógicas. Dentro de una amplia gama bibliográfica, se seleccionaron textos esenciales relacionados con el objeto de estudio.

\section{Programa de Didáctica Universitaria Interactiva.}

Con el objetivo general de contribuir a apoyar la práctica docente con herramientas actuales de la Didáctica universitaria, se valoró la puesta en práctica de un programa interactivo que combinara la presencialidad con el uso de recursos virtuales (UPS, GIEDIC, blog). La función mediadora del curso debía contribuir a lograr indicadores satisfactorios en cuanto a la preocupación de los docentes por el aprendizaje de todos los estudiantes, lograr una clase más explicativa y desarrolladora, así como intensificar el uso de recursos didácticos adecuados en el proceso de enseñanza aprendizaje.

El curso se concibió desde una metodología que combinara métodos y técnicas en el uso de los recursos de la información en la capacitación, privilegiando el intercambio profesional con las posibilidades del uso del Aula Virtual de Aprendizaje Cooperativo (AVAC) de la universidad. En particular, el AVAC, como el aula virtual brinda el acceso a los materiales de apoyo y profundización de los contenidos de cada curso. Es una metodología tendiente a reforzar el trabajo constructivista autónomo de los estudiantes. Igualmente se brindan consultas académicas en línea. Se pretendió posibilitar la socialización y la asimilación del contenido de forma cooperativa, prestando atención preferente a la vinculación teoría- práctica.

El grupo de docentes estaría integrado principalmente por ingenieros y licenciados de distintas carreras técnicas, económicas e informáticas, dedicados a la actividad pedagógica. Se hacía necesario sistematizar aspectos fundamentales de la Didáctica vinculados a los 
componentes del proceso de enseñanza aprendizaje, en particular las formas de organización derivadas de la clase universitaria. Los temas a desarrollar incluirían la introducción y el tratamiento de aspectos de profundización en torno a la clase universitaria, su planificación y momentos principales; los métodos activos de aprendizaje, el uso adecuado de medios didácticos, el trabajo cooperativo en grupos, así como la evaluación de los aprendizajes.

El curso se desarrollaría con una duración de 40 horas en total, a través de conferencias dialogadas, debates, trabajo grupal, trabajo colaborativo y trabajo autónomo. Esas formas de organización del proceso docente facilitan la socialización y asimilación del contenido de forma cooperada. Una atención preferente se otorgaría a la vinculación teoría - práctica para contribuir a perfeccionar las habilidades y competencias didácticas. La variedad de medios incluiría el uso del Entorno virtual, TICS, computadora, video beam, pizarra, papelógrafos, marcadores, libros y publicaciones periódicas.

El sistema de conocimientos se planificó desde la introducción, con la conceptualización de la Didáctica universitaria, los rasgos del proceso de enseñanza aprendizaje en la universidad, la educabilidad y la educatividad en la universidad, así como el rol del docente como mediador. En el tema 1 se abordaría elementos generales de la clase universitaria desde la planificación y momentos principales. Se tratarían aspectos tales como, el proyecto micro curricular del profesor, las características de cada uno de los componentes didácticos, los tipos de clase y su estructura, así como la motivación y el trabajo metodológico. Igualmente, se abordaría la temática relativa a la clase universitaria y su contextualización con la normativa vigente, mediante el trabajo en pequeños grupos y el debate de aspectos fundamentales. Para ello se apoyaría la exposición en la actividad práctica para la preparación de actividades docentes y la observación de video.

Por su parte, el tema 2 se concibió desde el tratamiento de los métodos activos de aprendizaje, desglosando el contenido en el reconocimiento de los conceptos de aprendizaje y enseñanza y su relación con su carácter activo. De esa manera se propiciaría el debate acerca de los métodos de activos, para enfocar algunos criterios acerca de los tipos, las estrategias de aprendizaje y la aplicación de los métodos en las diversas asignaturas y carreras desde el debate sobre sus particularidades.

El tema 3 se dedicaría al tratamiento de los medios didácticos, desde la clasificación y el enfoque de los recursos de la información científico en función de la docencia, en particular las posibilidades de la biblioteca como centro de recursos para el aprendizaje y el uso de catálogos y bibliotecas virtuales. Todo ello concluiría con una_actividad práctica en la biblioteca universitaria.

De igual manera, el tema 4 fue planificado desde el trabajo cooperativo en grupos, prestando atención preferente al aprendizaje grupal, desde la tipología y el reconocimiento de elementos del trabajo cooperativo en grupos y sus beneficios para el aprendizaje. Finalmente, concluiría con la aplicación del trabajo grupal en el aula. A su vez, el tema 5 estaría referido a la evaluación de los aprendizajes, partiendo del concepto de evaluación y sus particularidades como componente del proceso de enseñanza aprendizaje, los tipos de evaluación y su contextualización en la normativa vigente. 
El sistema de evaluación del curso se concibió para su realización en forma sistemática y continua, teniendo en cuenta la asistencia y puntualidad, las respuestas a preguntas en el desarrollo de las conferencias centrales, así como la participación en los talleres, el trabajo en equipos y las respuestas a las actividades enviadas por el tutor. En ese sentido, se destinarían12 horas para el trabajo autónomo, fundamentalmente para dar respuesta a actividades enviadas por el tutor, quien evaluaría a los participantes y asentaría las notas correspondientes. La evaluación final del curso y de los participantes preveía la presentación de un proyecto vinculado con la planificación del proceso docente de las asignaturas que imparten.

La ejecución del curso se produjo en las condiciones óptimas que ofrecen los locales de la Universidad Politécnica Salesiana, sede Guayaquil. Junto a elementos tradicionales como los papelógrafos y el pizarrón, se aprovecharon las posibilidades de proyección audiovisual, el acceso a Internet y en particular el AVAC, en el climatizado espacio áulico. Se combinaron formas expositivas con el debate, el aprendizaje grupal, la observación de videos y filmes y el trabajo autónomo en las diversas sesiones.

La planificación de las tareas docentes (T) incluyó acciones específicas a realizar en el AVAC, a partir de materiales de apoyo alojados en el espacio digital. Así, en la T1 se solicitó subir al AVAC la planificación curricular de una de las asignaturas a su cargo, teniendo en cuenta los elementos abordados en el curso sobre la Didáctica y el proyecto microcurricular del profesor. La T2 recabó subir al AVAC el cronograma de una de las asignaturas a su cargo, siguiendo las observaciones planteadas en el curso. Por su parte, la T3 requirió elaborar un informe valorativo de su asignatura dentro del currículo de la carrera, a partir de la relación entre ciencia y asignatura, el plan analítico, los componentes del proceso de enseñanza aprendizaje, los documentos normativos, la bibliografía y su experiencia como docente (en 5 páginas), que serían valoradas en el AVAC.

La T4 se dirigió a la elaboración de una relación de bibliografía de su asignatura, a partir de la consulta en el catálogo electrónico de la biblioteca de la UPS, señalando los datos de la referencia bibliográfica. El seminario(S) 1 incluyó el trabajo en grupos sobre el tema de aprendizaje grupal, estudio de material y elaboración de esquema lógico estructural. A su vez, el taller 3 se desarrolló mediante informes valorativos sobre la base de la observación de videos educativos elaborados por estudiantes. El S2 consistió en un seminario sobre la observación del filme "El profesor" y debate posterior. Finalmente, la T 5 se dirigió a realizar la autoevaluación.

\section{Resultados y discusión}

Los instrumentos de proceso aplicados durante la ejecución del curso permitieron evaluar sistemáticamente los avances en la transformación del aprendizaje de los docentes y el impacto en los modos de actuación de los participantes en la actividad de capacitación. A su vez, los resultados de la autoevaluación final aportaron importantes elementos para el análisis de la significación de la acción de capacitación ejecutada con el grupo de docentes participantes. Mediante la aplicación de un cuestionario se recopiló información valiosa que evidenció el salto cualitativo de los participantes, tanto en el plano teórico como en la práctica de su labor profesional. 
El $94 \%$ de los cursistas valoró el grado de cumplimiento de las tareas realizadas durante el curso en una escala de 1 a 10 puntos, en un puntaje entre 8 y 10. La evaluación de indicadores en las categorías prestablecidas, se manifestó en que la asistencia recibió $95 \%$ en la escala de excelente, mientras que la puntualidad fue del $80 \%$ en la categoría de excelente y $20 \%$ en la valoración de bien.

Otros indicadores se evaluaron de manera satisfactoria, pues el $80 \%$ considero excelente la participación en clase y de igual manera fue evaluado el trabajo en equipo. La entrega de tareas fue considerada como excelente por el $70 \%$ de los cursistas, mientras que el resto lo ubicó en la categoría de bien. Por su parte, la colaboración con otros compañeros recibió el $100 \%$ en la consideración de los encuestados, al igual que la disposición para asimilar lo tratado en el curso.

La valoración cualitativa de las opiniones en relación con los aprendizajes alcanzados en el curso aportó importantes referentes de análisis. La relación de elementos planteados por los encuestados incluyó un significativo grupo de aspectos como la redacción de objetivos, metodologías y plan, "dejar de satanizar el uso de la tecnología dentro de las clases”. También la mejora en la elaboración de la planificación de cada una de las actividades docentes, conocimientos sobre cómo se debe dictar una clase, la organización del contenido de una asignatura, así como las técnicas y metodologías que se deben aplicar.

Otros aprendizajes según los encuestados, se manifestaron en que se llegó a la conclusión de que el profesor es el facilitador y guía de ese proceso educativo en la clase y que debe seguir un proceso pedagógico utilizando todas las herramientas metodológicas posibles. De igual manera, se expresó la idea de la importancia de conocer las bases científicas de los componentes fundamentales del enfoque tradicional de la Didáctica y cómo se debe planificar la clase, teniendo en cuenta los objetivos y el nivel de aprendizaje que deben adquirir los estudiantes.

Los planteamientos de los docentes participantes en el curso, expresaron su complacencia con el esclarecimiento de cómo aplicar los diferentes tipos de clases, así como el perfeccionamiento en la planificación de las actividades de los estudiantes y los tiempos durante el desarrollo de la actividad áulica. También se manifestaron opiniones sobre la utilización correcta de los verbos que denoten acción al momento de confeccionar los objetivos de la asignatura, así como la aplicación de métodos y su importancia.

Para los docentes, el curso les permitió enriquecer su labor pedagógica en aspectos tan significativos como la relación entre los objetivos y el contenido de la asignatura. Se reconoció que desde el punto de vista vigotskiano, el estudiante debe ser el centro y objetivo del proceso enseñanza-aprendizaje. Finalmente se consideró que el curso contribuyó a que los participantes puedan mejorar la planificación de la estructura de las clases.

Ante la interrogante sobre las dificultades encontradas para aprender en el curso, el $40 \%$ de los docentes expresó que no existieron limitaciones y todas las temáticas abordadas fueron suficientemente esclarecidas. Otros cursistas señalaron que el tiempo asignado al curso pudiera 
ampliarse y que este tipo de espacio de intercambio profesional debería ser permanente, de modo que la actualización en este sentido fuera más sistemática.

La pregunta ¿Qué aspectos te gustaron más?, fue respondida con diversas respuestas por los participantes en el curso. Se consideró la redacción de objetivos, la forma de llevar el seminario, conectando los temas en forma fluida y adecuada; la interacción entre docente alumno y entre compañeros en el desarrollo del curso, así como la participación y buena predisposición de compañeros y el profesor. Otros docentes se refirieron a la organización, el trabajo en grupo y su aporte, así como aprender métodos de aprendizaje y herramientas pedagógicas. También se resaltó la aplicación de diversas dinámicas grupales.

A uno de los participantes le gustó que el módulo de capacitación efectuado se hubiera planificado de esa manera. "Primero me gusto porque como docente también hago lo mismo, planifico todo cronológicamente en mi asignatura, para no tener contratiempos sobre qué es lo que voy a dar en cada clase y segundo me gustó esa facilidad, que a manera de mensaje, llegaba hasta nosotros indicándonos la forma y la responsabilidad de nuestra labor como docentes".

A un cursista le gustó que se tratara la temática del análisis metodológico de la asignatura y sus contenidos, relacionándola con la ciencia a la cual pertenece. También agradaron las actividades académicas en grupo, que el curso fuera muy motivador e interesante y la retroalimentación alcanzada entre los diferentes docentes, pues a medida que se desarrollaban los temas, varios profesores compartieron los aspectos que les habían resultado positivos en sus clases y cuáles no.

Entre los aspectos que satisficieron a los docentes estuvo la explicación de cómo realizar un plan analítico y su importancia, así como también el lenguaje sencillo, sin caer demasiado en tecnicismos y abuso de referencias a autores de tal o cual teoría de docencia. Un profesor manifestó que este fue un curso más práctico, en el sentido que iba acompañado de anécdotas y vivencias, tanto del profesor como de los alumnos.

"Me gustaron las recomendaciones y la repetitividad para alcanzar la comprensión y memorización de los participantes", según un participantes. Para otro docente, fue satisfactoria la metodología empleada por el capacitador y los métodos aplicados. Fue interesante, según las opiniones expresadas, el compañerismo y la alegría reinante así como la confianza generada por el docente en la actividad.

Otros aspectos incluyeron la disposición de varias actividades para la evaluación del proceso y la utilización del AVAC como herramienta complementaria. "Me gustó sentir que el profesor es un actor que en su entorno personal tiene un rol que es valioso para la sociedad. No es sólo es un sujeto que enseña, sino que es un ser humano que tiene la importante tarea de influenciar de manera positiva en los jóvenes, a pesar de las limitaciones que posean”, expresó uno de los participantes.

De esa manera, el 94 \% consideró que este curso contribuyó a variar sus ideas o puntos de vista sobre el proceso de enseñanza aprendizaje. El aspecto modificado en sus concepciones pedagógicas reflejó una amplia relación de opiniones, que incluyó el mejoramiento de la 
preparación de clases, el esclarecimiento de la forma de llegar al estudiante y la evaluación de una asignatura y su importancia. También se modificó sus puntos de vista sobre los métodos para dirigir el proceso de enseñanza aprendizaje y considerar que el centro del proceso pedagógico es el estudiante por lo que es necesario contar con herramientas didácticas adecuadas para motivar su aprendizaje. Igualmente se señaló que lo más importante de educar es preparar al estudiante para la vida, porque el futuro de la sociedad está en ese joven.

Otras ideas que variaron en los docentes se refieren a las técnicas pedagógicas requeridas para cada actividad. "Ahora tengo muy claro la forma de como planificar la clase porque, yo mismo fui el que durante una clase hizo la diferencia entre el plan de actividades que actualmente llenamos en la carrera y el plan de actividades de clase, que se estaba proponiendo en las plenarias de cada grupo según el tema que se les asigno el tutor y llegué a la conclusión de que esa era una mejor propuesta".

"El curso estuvo muy claro y preparado con una buena metodología, no fue aburrido. Más bien fue ameno y divertido con las técnicas utilizadas, incluido el llamado amigo secreto". Se demostró lo significativo de la bibliografía actualizada dentro del plan curricular y lo que puede incidir positivamente en el proceso enseñanza-aprendizaje.

Para un participante resultó importante profundizar en la relación ciencia-asignatura y que las actividades en la clase sean diseñadas de una forma que acerque al estudiante a los principales temas de las ciencias de donde se derivan las asignaturas. De esa manera podrá lograrse mayor nivel de interés en el estudiante para su formación en el plano científico y tecnológico.

\section{Conclusiones}

En resumen, la experiencia puesta en práctica en la UPS posibilitó la interacción en el proceso de capacitación didáctica de los profesionales, a través de acciones docentes presenciales o virtuales. Con ello se propició la elevación de los niveles de responsabilidad de los profesionales para lograr una labor académica de rigor sin descuidar el ángulo educativo del proceso de formación del estudiante.

La Didáctica puede ocupar un lugar central en la capacitación de los docentes de la Educación Superior, atendiendo a las particularidades de ese nivel de enseñanza y las exigencias que plantea la realidad actual a las instituciones educacionales.

La utilización combinada de tecnologías virtuales y recursos de la información en la capacitación, favorece las condiciones para lograr el aprendizaje efectivo en los profesionales universitarios. En ese ámbito se resalta la significación de la labor didáctica para concretar los vínculos entre la ciencia y la asignatura con el fin de contribuir a la formación integral de los estudiantes, en el marco de los procesos sustantivos de la institución de Educación Superior.

Las recomendaciones de los docentes participantes se expresaron, en síntesis, en la necesidad de una preparación continua. “... si bien tenemos un título profesional, debemos capacitarnos en Pedagogía, Didáctica, Andragogía, pues el maestro no sólo debe serlo sino 
también parecerlo". Para coadyuvar en esa capacitación se hizo saber la propuesta de que a nivel de universidad exista una estructura especializada en temas de Didáctica, que se dedique a la formación permanente de los docentes a través de talleres, conferencias y elaborar guías, entre otros aspectos.

En el plano valorativo se contribuyó a reafirmar el sentido de pertenencia hacia la profesión pedagógica, con independencia de las asignaturas que se dirijan en el proceso de enseñanza aprendizaje. La mayoría de los participantes manifestó el impacto positivo del curso en la transformación de su docencia.

\section{Bibliografía}

Alzate P, María Victoria., Miguel Ángel Gómez M. [y] Martha Cecilia Arbeláez. Enseñar en la universidad: saberes, prácticas y textualidad. Bogotá: Ecoe Ediciones, 2011.

Addine Fernández, Fátima. Didáctica: teoría y práctica. Ed. Pueblo y Educación. Ciudad de la Habana, 2004.

Bowden, John y Reference Marton. La universidad un espacio para el aprendizaje. Madrid: Narcea, 2012.

Brown, Sally y Ruth Pickford. Evaluación de habilidades y competencias en Educación Superior. Madrid: Narcea, 2013.

Chávez Rodríguez, Justo; Amparo Suárez Lorenzo, Luis Daniel Permuy González.

Acercamiento necesario a la pedagogía general. Editorial Pueblo y Educación, La Habana, Cuba, 2005.

Comenio,JuanAmos.DidácticaMagna.sb8f45ce1279fc46e.jimcontent.com/download/.../DIDÁCT ICA\%20MAGNA.pptx

Plan Nacional del Buen Vivir. Ecuador. http://www.buenvivir.gob.ec/inicio

García-Valcárcel Muñoz, Ana. Integración de las TIC en la docencia universitaria: situación actual y retos en el EEES. Repiso. -- (La Cataluña): Netbiblo, 2011.

González Rivera, Pedro Luis. Propuesta de curso de actualización docente.2016 http://virtual.ups.edu.ec/paracademico48/course/view.php?id=1771

González Rivera, P.L.; Hernández Rodríguez, D. Educación para el emprendimiento.

Revista Mendive, No. 52, $2015 . \quad$ http://mendive.upr.edu.cu/index.php/todas/no-52/556articulos/1097-pedro-luis-gonzalez-rivera 
Hernández, Deogracia y Pedro Luis González. Educabilidad, emprendimiento e investigación en la formación de los profesionales. Ponencia en Congreso Pedagogía 2017, Universidad de Pinar del Río, Cuba, 2016.

Instituto Central de Ciencias Pedagógicas de Cuba, 1999. http://didacticadidactico.blogspot.com/2014/02/instituto-central-de-ciencias.html

Klingberg, Lothar. Introducción a la Didáctica General. Ed. Pueblo y Educación. Ciudad de la Habana, 1972.

Labarrere Reyes, Guillermina y Valdivia Pairol, Gladys E. Pedagogía. Editorial Pueblo y Educación, La Habana, 1988.

Luengo Navas, Julián. La educación como hecho. http://www.ugr.es/ fjjrios/pce/media/1EducacionHecho.pdf

Martí Pérez, José. Obras Completas. Editorial de Ciencias Sociales, La Habana, Tomo 8.

Moncada, Ligia. Reflexiones sobre la educación universitaria: didáctica. -- Bogotá: Universidad Nacional de Colombia, 2009.

Nassif, Ricardo. Pedagogía general. Buenos Aires, Kapelusz, 1974.https://es.scribd.com/doc/138181089/Ricardo-Nassif-Pedagogia-General-Cap-1

Pezo Ortiz, Elsa. Didáctica general. Quito: Codeu, 2010.

Padrón, Carmen; González Rivera, P. L.; Hernández Rodríguez, D. y González Hernández, M. Los recursos de la información para la investigación en la universidad. Rev. Ciencias Médicas [online].2015, vol.19, No.3, pp.578-588. Disponible en: $<$ http://scielo.sld.cu/scielo.php?script=sci_arttext\&pid=S156131942015000300021\&lng=es\&nrm=iso>. ISSN 1561-3194.

Sánchez González, Ma. Paz. Técnicas docentes y sistemas de evaluación en educación superior. Madrid: Narcea, 2010.

Sevillano García, María Luisa (Coord.). Medios, recursos didácticos y tecnología educativa. Madrid: Pearson, 2011.

UNESCO, Declaración de Incheon. http://es.unesco.org/world-education-forum-2015/aboutforum/declaracion-de-incheon

UPS, GIEDIC, blog. http://giedic.blog.ups.edu.ec/curso-didactica-universitaria/. 